\title{
A NON-EXTREME-POINTS APPROACH TO EXTREME POINTS OF INTEGRAL FAMILIES OF ANALYTIC FUNCTIONS
}

\author{
K. Dow \\ (Received 4 October, 2018)
}

\begin{abstract}
Non extreme points of compact, convex integral families of analytic functions are investigated. Knowledge about extreme points provides a valuable tool in the optimization of linear extremal problems. The functions studied are determined by a 2-parameter collection of kernel functions integrated against measures on the torus. Families from classical geometric function theory such as the closed convex hull of the derivatives of normalized close-to-convex functions, the ratio of starlike functions of different orders, as well as many others are included. However for these families of analytic functions, identifying "all" the extreme points remains a difficult challenge except in some special cases. Aharonov and Friedland [1] identified a band of points on the unit circle which corresponds to the set of extreme points for these 2-parameter collections of kernel functions. Later this band of extreme points was further extended by introducing a new technique by Dow and Wilken [3]. On the other hand, a technique to identify a non extreme point was not investigated much in the past probably because identifying non extreme points does not directly help solving the optimization of linear extremal problems. So far only one point on the unit circle has been identified which corresponds to a non extreme point for a 2-parameter collections of kernel functions. This leaves a big gap between the band of extreme points and one non extreme point. The author believes it is worth developing some techniques, and identifying non extreme points will shed a new light in the exact determination of the extreme points. The ultimate goal is to identify the point on the unit circle that separates the band of extreme points from non extreme points. The main result introduces a new class of non extreme points.
\end{abstract}

\section{Preliminaries and Statement of Main Theorem}

Let $\mathbb{D}$ and $\Gamma$, respectively, denote the open unit disk and the unit circle in the complex plane $\mathbb{C}$. Let $H(\mathbb{D})$ denote the space of functions analytic in $\mathbb{D}$ and let $\mathbb{T}=\Gamma \times \Gamma$ denote the torus. We investigate non extreme points of the compact convex families in $H(\mathbb{D})$ defined by, for $p, q>0$,

$$
F_{p, q}=\left\{f_{\mu}(z)=\int_{\mathbb{T}} \frac{(1-x z)^{p}}{(1-y z)^{q}} d \mu(x, y) \mid \mu \text { is a probability measure on } \mathbb{T}\right\} .
$$

A function $f \in F_{p, q}$ is an extreme point of $F_{p, q}$ if $f=t g+(1-t) h$ for some $g, h \in F_{p, q}$ and $0<t<1$, then $f=g=h$. If a function $f \in F_{p, q}$ is not an extreme point, then it is a non extreme point of $F_{p, q}$. For particular choices of the parameters, $F_{p, q}$ includes the closed convex hull of the derivatives of the normalized closed-to-convex functions on $\mathbb{D}[\mathbf{2}]$, the ratio of starlike functions of different orders 
[1], as well as many other families from classical geometric function theory [4], [5]. In the search for non extreme points of $F_{p, q}$, our particular choice of probability measures in this paper captures the known non extreme points [1] as we expected, but more excitingly it leads to the existence of, for given $p$ and $q$, the non extreme points of $F_{p, q}$ nearby when $p$ is a positive integer.

Consider the curve $C_{p}=\left\{(1-x)^{p}:|x|=1\right\}$ and let $E_{p}$ denote the closed convex hull of $C_{p}$ in $\mathbb{C}$. The following are well-known facts:

(i) If there exists a probability measure $\mu$ that is not a unit point mass and representing a function $f(z)=f_{\mu}(z)$, then $f$ is a non extreme point of $F_{p, q}$ [2].

(ii) The family $F_{p, q}$ is closed under rotation. That is, if $f$ is a function in $F_{p, q}$ and $|u|=1$, then $g(z)=f(u z)$ is also in $F_{p, q}$. Thus, any rotation of a non extreme point is a non extreme point. For a simpler computation, we will show $\frac{(1-x z)^{p}}{(1-z)^{q}}$ is a non extreme point for some $x \in \Gamma$.

(iii) (The Product Theorem) Suppose that $\alpha>0$ and $F_{\alpha}$ denotes the set of functions represented by

$$
f(z)=\int_{\Gamma} \frac{1}{(1-y z)^{\alpha}} d \mu(y)
$$

where $\mu$ is a probability measure on the unit circle. If $f \in F_{\alpha}, g \in F_{\beta}, \alpha>0$ and $\beta>0$, then $f g \in F_{\alpha+\beta}[\mathbf{2}]$.

(iv) For $0<p \leq q, \frac{(1-x z)^{p}}{(1-z)^{q}}$ is a non extreme point of $F_{p, q}$ when $x=1$ by the Product Theorem. Then by (ii), $\frac{(1-x z)^{p}}{(1-y z)^{q}}$ is a non extreme point of $F_{p, q}$ when $x=y$ for $0<p \leq q$.

(v) If $x \neq 1$ and $(1-x)^{p}$ is an extreme point of $E_{p}$, then $\frac{(1-x z)^{p}}{(1-z)^{q}}$ is an extreme point of $F_{p, q}[\mathbf{1}]$.

(vi) For $0<p \leq 1$, the curve $C_{p}$ encloses a convex region and every point on $C_{p}$ is an extreme point of $E_{p}$. In this case, by (v), the only possible non extreme point of $F_{p, q}$ is $\frac{(1-x z)^{p}}{(1-z)^{q}}$ where $x=1$.

(vii) For $p>1$, as one traverses the curve $C_{p}$ in either direction starting at $2^{p}$ $(x=-1)$ and ending up at the origin $(x=1)$, there are two distinguished "turning points". These turning points occur when $|\arg (x)|=\pi(p-1) /(p+1)$ and correspond to the points on $C_{p}$ where $\operatorname{Re}(1-x)^{p}$ attains its minimum value. In this case, the convex set $E_{p}$ is bounded by part of the curve $C_{p}$ - the part traversed from one turning point through $2^{p}$ to the other turning point - together with the vertical line segment joining the two turning points. Thus, if $p>1$, a point $(1-x)^{p}$ is an extreme point of $E_{p}$ if and only if $\pi(p-1) /(p+1) \leq|\arg (x)| \leq \pi$. Then by $(\mathrm{v})$, the only possible non extreme points of $F_{p, q}$ are $\frac{(1-x z)^{p}}{(1-z)^{q}}$ where $0 \leq|\arg (x)|<\pi(p-1) /(p+1)[\mathbf{3}]$.

Two fundamental questions arise. The first question is whether $\frac{(1-x z)^{p}}{(1-z)^{q}}$ is a non extreme point when $x=1$ for any $p>0$, and $q>0$. The second question addresses the existence of non extreme points $\frac{(1-x z)^{p}}{(1-z)^{q}}$ near $x=1$. In this paper, we address both of these questions for a special case when $p=2,3,4, \ldots$ and $q \geq 1$. We are 
now ready to state the main result.

Theorem 1.1 (Main Theorem). For $p=n=2,3,4, \ldots$ and $q \geq 1, \frac{(1-x z)^{n}}{(1-z)^{q}}$ is a non extreme point of $F_{n, q}$ when $x=1$. Moreover, there exists some non extreme points near $x=1$.

In the proof of the existence of non extreme points $\frac{(1-x z)^{p}}{(1-z)^{q}}$ near $x=1$, how close $x$ is to $y$ (in this case, $y=1$ ) plays an important role which you will see towards the end of the proof.

\section{Proof of Main Theorem}

We begin the proof of Theorem1.1 by showing $\frac{(1-x z)^{n}}{(1-z)^{q}}$ is a non extreme point of $F_{n, q}$ when $x=1$.

Remark 2.1. Recall the Fejer kernel

$$
k_{n}(x)=1+2 \sum_{k=1}^{n-1} \frac{n-k}{n} \cos (k x)=\frac{1}{n}\left[\frac{1-\cos (n x)}{1-\cos (x)}\right] .
$$

Note that $k_{n}(x)$ is non-negative and has zeros at $x=\frac{2 \pi j}{n}$, where $j=1,2,3, \ldots$ $(n-1)$. Let

$$
d \lambda=\left[1+2 \sum_{k=1}^{n-1} \frac{n-k}{n} \cos (k \theta)\right] \frac{d \theta}{2 \pi} .
$$

Then $\lambda$ is a non-negative measure with total mass of 1 over $\Gamma$.

Remark 2.2. By the Product Theorem, for $q \geq 1$ there exists a probability measure $\nu$ over the unit circle which is not a unit point mass, and satisfies

$$
\frac{1}{(1-z)^{q-1}}=\int_{\Gamma} \frac{1}{(1-y z)^{q}} d \nu(y) \text {. }
$$

Proof of the first half of Theorem 1.1. Let $\mu$ be a product measure $\mu=\lambda \times \nu$ over the $\mathbb{T}$ where $\lambda$ and $\nu$ are in Remarks 2.1 and 2.2, respectively. Then,

$$
\begin{aligned}
f_{\mu}(z) & =\int_{\mathbb{T}} \frac{(1-x z)^{n}}{(1-y z)^{q}} d \mu \\
& =\int_{\Gamma} \int_{\Gamma} \frac{(1-x z)^{n}}{(1-y z)^{q}} d \lambda(x) \times d \nu(y) \\
& =\frac{1}{(1-z)^{q-1}} \int_{\Gamma}(1-x z)^{n} d \lambda(x) \\
& =\frac{1}{(1-z)^{q-1}} \int_{0}^{2 \pi}\left(1-e^{i \theta} z\right)^{n}\left[1+2 \sum_{k=1}^{n-1} \frac{n-k}{n} \cos (k \theta)\right] \frac{d \theta}{2 \pi} \\
& =\frac{1}{(1-z)^{q-1}}\left[\sum_{k=0}^{n-1}(-1)^{k} \frac{(n-1) !}{(n-1-k) ! k !} z^{k}\right]
\end{aligned}
$$




$$
\begin{aligned}
& =\frac{1}{(1-z)^{q-1}} \cdot(1-z)^{n-1} \\
& =\frac{(1-(1) z)^{n}}{(1-z)^{q}}
\end{aligned}
$$

Therefore by (i), $\frac{(1-x z)^{n}}{(1-z)^{q}}$ is a non extreme point when $x=1$.

Before we prove the second half of Theorem 1.1, that is: for $p=n=2,3,4, \ldots$ and $q \geq 1$, there exists some non extreme points $\frac{(1-x z)^{p}}{(1-z)^{q}}$ of $F_{n, q}$ when $x$ is near 1 , we need to define the following two probability measures; $\mu_{1}$ on $\Gamma \times\{1\}$ and $\mu_{2}$ on $\Gamma \times(\Gamma \backslash\{1\})$. As mentioned in (vii), turning points of $C_{n}$ occur when $\left|\arg \left(x_{0}\right)\right|=$ $\frac{\pi(n-1)}{(n+1)}$. Without loss of generality, let $x_{0}=e^{i \theta_{0}}, 0<\theta_{0}<\pi$ (because of the symmetry of $C_{n}$, the following argument works for $-\pi<\theta_{0}<0$ similarly). Let $\alpha$ be an angle such that $\theta_{0}<\alpha \leq \frac{\pi(n-1)}{(n+1)}$, and $\delta_{e^{i \alpha}}, \delta_{e^{-i \alpha}}$ be unit point masses at $e^{i \alpha}$ and $e^{-i \alpha}$ respectively. We now define a probability measure $\mu_{1}$ on $\Gamma \times\{1\}$ as

$$
\mu_{1}=R \delta_{e^{i \alpha}}+(1-R) \delta_{e^{-i \alpha}} \text { for some } 0<R<1 .
$$

Then,

$$
\int_{\Gamma \times\{1\}}(1-x)^{n} d \mu_{1}=R\left(1-e^{i \alpha}\right)^{n}+(1-R)\left(1-e^{-i \alpha}\right)^{n}
$$

which is a point on the line segment connecting the two points; $\left(1-e^{i \alpha}\right)^{n}$ and $\left(1-e^{-i \alpha}\right)^{n}$ on $C_{n}$. We also define

$$
\mu_{2}=\lambda(x) \times \nu(y) \text { on } \Gamma \times(\Gamma \backslash\{1\}) \text { where } \frac{1}{(1-z)^{q-1}}=\int_{\Gamma} \frac{1}{(1-y z)^{q}} d \nu(y) .
$$

Again, the existence of such a probability measure $\nu$ is guaranteed by (iii). We will discuss the details about the probability measure $\lambda$ in the proof below.

Proof of the second half of Theorem 1.1. Let

$$
\mu=\left\{\begin{array}{l}
\epsilon \cdot \mu_{1} \text { on } \Gamma \times\{1\} \\
(1-\epsilon) \cdot \mu_{2} \text { on } \Gamma \times(\Gamma \backslash\{1\})
\end{array}\right.
$$

for some $0<\epsilon<1$. Then for some $x_{0}$ near 1 ,

$$
\begin{aligned}
\frac{\left(1-x_{0} z\right)^{n}}{(1-z)^{q}} & =\int_{\mathbb{T}} \frac{(1-x z)^{n}}{(1-y z)^{q}} d \mu \\
& =\epsilon \int_{\Gamma \times\{1\}} \frac{(1-x z)^{n}}{(1-z)^{q}} d \mu_{1}+(1-\epsilon) \int_{\Gamma \times(\Gamma \backslash\{1\})} \frac{(1-x z)^{n}}{(1-y z)^{q}} d \mu_{2} .
\end{aligned}
$$

The probability measure $\mu$ in (2.3) has three components; $\epsilon, \mu_{1}$ and $\mu_{2}$. If we can successfully describe this probability measure $\mu$ which satisfies equation (2.4), then we prove the second half of Theorem 1.1. The rest of the proof will discuss the conditions that $\epsilon, \mu_{1}$ and $\mu_{2}$ must satisfy in order for (2.4) to hold, and the existence of such probability measures. My original idea was that the probability measure $\mu$ we need in order to show $\frac{\left(1-x_{0} z\right)^{p}}{(1-z)^{q}}$ is a non extreme point when $x_{0}$ is near 1 must be similar to the probability measure we used for $x_{0}=1$ earlier. Therefore we will 
examine how $\mu$ and its three components; $\epsilon, \mu_{1}$ and $\mu_{2}$ behave as $x_{0} \rightarrow 1$ in order to construct a desirable $\mu$.

First, we will show that $\epsilon$ in the probability measure $\mu$ described in (2.3) approaches 0 as $x_{0} \rightarrow 1$. Rearrange the equation $(2.4)$ by multiplying $(1-z)^{q}$ on both sides, and let $z$ approach 1 radially from $\mathbb{D}$. Then by the Lebesgue Dominated Convergence Theorem, we obtain

$$
\left(1-x_{0}\right)^{n}=\epsilon \int_{\Gamma \times\{1\}}(1-x)^{n} d \mu_{1} .
$$

With the choice of $\mu_{1}$ described in (2.1),

$$
\begin{aligned}
\left(1-x_{0}\right)^{n} & =\epsilon\left[R\left(1-e^{i \alpha}\right)^{n}+(1-R)\left(1-e^{-i \alpha}\right)^{n}\right] \\
& =\epsilon\left[R\left(1-e^{i \alpha}\right)^{n}+(1-R)\left(1-e^{-i \alpha}\right)^{n}\right]+(1-\epsilon) \cdot 0 .
\end{aligned}
$$

Hence the point $\left(1-x_{0}\right)^{n}$ on $C_{n}$ lies on the line segment connecting a point $R\left(1-e^{i \alpha}\right)^{n}+(1-R)\left(1-e^{-i \alpha}\right)^{n}$ and the origin for an appropriate choice of $R$ (see Figure 1).

For a chosen angle $\alpha$ in $\mu_{1}$ from (2.1), the points $A$ and $B$ were determined. For a given $x_{0}$ near 1 , the point $D$ is determined. We connect the point $D$ and the origin then extend it to find the point $C$. This will determine $\epsilon$ since $D=$ $\epsilon \cdot C+(1-\epsilon) \cdot 0$. As $x_{0}$ approaches 1 on the unit circle (i.e, $\theta_{0} \downarrow 0$ for $x_{0}=e^{i \theta_{0}}$ ), the point $D=\left(1-x_{0}\right)^{n}$ approaches the origin. Therefore as $x_{0}$ approaches $1, \epsilon \rightarrow 0$.

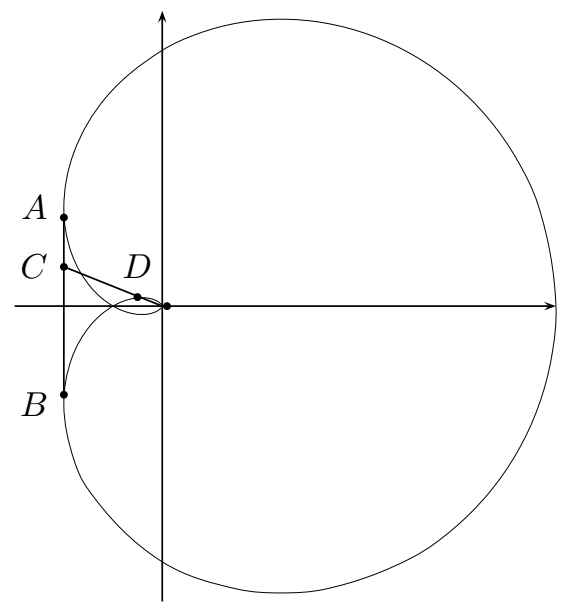

Figure 1: picture of $C_{n}=\left\{(1-x)^{p}:|x|=1\right\}$ with $A=\left(1-e^{-i \alpha}\right)^{n}, B=$ $\left(1-e^{i \alpha}\right)^{n}, C=R\left(1-e^{i \alpha}\right)^{n}+(1-R)\left(1-e^{-i \alpha}\right)^{n}$, and $D=\left(1-x_{0}\right)^{n}$.

Recall the probability measures $\mu_{1}$ and $\mu_{2}$ described in (2.1) and (2.2). Now we examine the relationship between $\mu_{1}$ and $\lambda$. First if we expand the equation (2.5), we obtain

$$
\epsilon \sum_{k=0}^{n}\left(\begin{array}{l}
n \\
k
\end{array}\right)(-1)^{k} \int_{\Gamma \times\{1\}} x^{k} d \mu_{1}=\sum_{k=0}^{n}\left(\begin{array}{l}
n \\
k
\end{array}\right)(-1)^{k} x_{0}^{k} .
$$


Thus

$$
1-\epsilon=\sum_{k=1}^{n}\left(\begin{array}{l}
n \\
k
\end{array}\right)(-1)^{k+1}\left[x_{0}^{k}-\epsilon \int_{\Gamma \times\{1\}} x^{k} d \mu_{1}\right] .
$$

Manipulate the equation (2.4) to yield

$$
\begin{aligned}
& (1-\epsilon) \int_{\Gamma \times(\Gamma \backslash\{1\})} \frac{(1-x z)^{n}}{(1-y z)^{q}} d \mu_{2} \\
= & \frac{\left(1-x_{0} z\right)^{n}-\epsilon \int(1-x z)^{n} d \mu_{1}}{(1-z)^{q}} \\
= & \frac{1-\epsilon+\sum_{k=1}^{n}\left(\begin{array}{l}
n \\
k
\end{array}\right)(-1)^{k}\left[x_{0}^{k}-\epsilon \int x^{k} d \mu_{1}\right] z^{k}}{(1-z)^{q}} .
\end{aligned}
$$

Then substitute the equation (2.6) for $1-\epsilon$ on the right-hand side above, we obtain

$$
\begin{aligned}
& (1-\epsilon) \int_{\Gamma \times(\Gamma \backslash\{1\})} \frac{(1-x z)^{n}}{(1-y z)^{q}} d \mu_{2} \\
= & \frac{\sum_{k=1}^{n}\left(\begin{array}{l}
n \\
k
\end{array}\right)(-1)^{k+1}\left[x_{0}^{k}-\epsilon \int x^{k} d \mu_{1}\right]\left(1-z^{k}\right)}{(1-z)^{q}} \\
= & \frac{\sum_{k=1}^{n}\left(\begin{array}{l}
n \\
k
\end{array}\right)(-1)^{k+1}\left[x_{0}^{k}-\epsilon \int x^{k} d \mu_{1}\right](1-z)\left(1+z+z^{2}+\ldots+z^{k-1}\right)}{(1-z)^{q}} \\
= & \frac{\sum_{k=1}^{n}\left(\begin{array}{l}
n \\
k
\end{array}\right)(-1)^{k+1}\left[x_{0}^{k}-\epsilon \int x^{k} d \mu_{1}\right]\left(1+z+\ldots+z^{k-1}\right)}{(1-z)^{q-1}} .
\end{aligned}
$$

Now if we apply $\mu_{2}$ described in (2.2) to the left-hand side, the expression becomes

$$
\begin{aligned}
\frac{(1-\epsilon)}{(1-z)^{q-1}} & \int_{\Gamma}(1-x z)^{n} d \lambda(x) \\
& =\frac{\sum_{k=1}^{n}\left(\begin{array}{l}
n \\
k
\end{array}\right)(-1)^{k+1}\left[x_{0}^{k}-\epsilon \int x^{k} d \mu_{1}\right]\left(1+z+\ldots+z^{k-1}\right)}{(1-z)^{q-1}} .
\end{aligned}
$$

Hence we obtain

$$
\begin{aligned}
(1-\epsilon) & \int(1-x z)^{n} d \lambda(x) \\
& =\sum_{k=1}^{n}\left(\begin{array}{l}
n \\
k
\end{array}\right)(-1)^{k+1}\left[x_{0}^{k}-\epsilon \int x^{k} d \mu_{1}\right]\left(1+z+\ldots+z^{k-1}\right) .
\end{aligned}
$$

The equation (2.7) shows the relationship between $\mu_{1}$ and $\lambda$. By comparing the coefficient of $z^{k}$, we gather the following $n+1$ conditions.

$$
\begin{aligned}
& (1-\epsilon) \frac{n !}{(n-i+1) !(i-1) !} \int(-1)^{i-1} x^{i-1} d \lambda \\
& \quad=\sum_{j=i}^{n}(-1)^{j+1} \frac{n !}{(n-j) ! j !}\left(x_{0}^{j}-\epsilon \int x^{j} d \mu_{1}\right) \text { for } 1 \leq i \leq n
\end{aligned}
$$


and

$$
\int x^{n} d \lambda=0
$$

The above conditions describe the specifics properties $\lambda$ has to satisfy. Now we describe how $\lambda$ must behave as $x_{0}$ approaches 1 .

Let

$$
U_{l}+i V_{l}=\frac{l}{(n-l+1)(1-\epsilon)}\left(x_{0}^{n-l+1}-\epsilon \int x^{n-l+1} d \mu_{1}\right)
$$

for some $U_{l}, V_{l} \in \mathbb{R}, \sqrt{U_{l}^{2}+V_{l}^{2}} \leq 1$.

Since $\epsilon \rightarrow 0$ as $x_{0}$ approaches 1 on the unit circle (i.e, $\theta_{0} \downarrow 0$ for $x_{0}=e^{i \theta_{0}}$ ), $U_{l}+i V_{l} \rightarrow \frac{l}{n-l+1}$ as $x_{0}$ approaches 1 . Now consider $k+1^{\text {st }}$ condition above.

Then,

$$
\int x^{k} d \lambda=\sum_{i=1}^{n-k}(-1)^{i+1} \frac{(n-k) ! k !}{(n-k-i+1) !(k+i-1) !}\left(U_{n-k-i+1}+i V_{n-k-i+1}\right) .
$$

Therefore as $x_{0}$ approaches 1 ,

$$
\begin{aligned}
\int x^{k} d \lambda \rightarrow & \sum_{i=1}^{n-k}(-1)^{i+1} \frac{(n-k) ! k !}{(n-k-i+1) !(k+i-1) !} \cdot \frac{n-k-i+1}{(k+i+1)(k+i)} \\
& =\frac{n-k}{n}, \text { for } 1 \leq k \leq n-1 .
\end{aligned}
$$

Let $A_{k}=\operatorname{Re} \int x^{k} d \lambda$ and $B_{k}=\operatorname{Im} \int x^{x} d \lambda$. Then $A_{k} \rightarrow \frac{n-k}{n}$ and $B_{k} \rightarrow 0$ as $x_{0}$ approaches 1 . Now we propose the following measure $d \lambda_{x_{0}}$ on $\Gamma$ which behaves as $\lambda$. Define

$$
d \lambda_{x_{0}}=\left[1+2 \sum_{k=1}^{n-1}\left(A_{k} \cos (k \theta)+i B_{k} \sin (k \theta)\right)\right] \frac{d \theta}{2 \pi}
$$

Then,

(a) $\int d \lambda_{x_{0}}=1$

(b) $\int x^{k} d \lambda_{x_{0}}=A_{k}+i B_{k}$ for $1 \leq k \leq n-1$

(c) $\int x^{n} d \lambda_{x_{0}}=0$.

It is important to point out that $d \lambda_{x_{0}}$ is not guaranteed to be non-negative. However, its limit is non-negative, and it satisfies the above three conditions.

$$
\lim _{x_{0} \rightarrow 1} d \lambda_{x_{0}}=\left[1+2 \sum_{k=1}^{n-1} \frac{n-k}{n} \cos (k \theta)\right] \frac{d \theta}{2 \pi}
$$

Notice that $\left[1+2 \sum_{k=1}^{n-1} \frac{n-k}{n} \cos (k \theta)\right] \frac{d \theta}{2 \pi}$ is the probability measure which was used to prove that $\frac{\left(1-x_{0} z\right)^{n}}{(1-z)^{q}}$ is a non extreme point when $x_{0}=1$ earlier. Moreover, $\left[1+2 \sum_{k=1}^{n-1} \frac{n-k}{n} \cos (k \theta)\right] \frac{d \theta}{2 \pi}=\frac{1}{n}\left[\frac{1-\cos (n \theta)}{1-\cos (\theta)}\right] \frac{d \theta}{2 \pi}$ has zeros at $\theta=\frac{2 \pi k}{n}$ where $k=$ $1,2, \ldots, n-1$, and each zero is $\frac{2 \pi}{n}$ apart. What we need to finish the proof now is to define a measure which behaves as (2.8), and guaranteed to be non-negative over $\Gamma$. To achieve this, we modify (2.8) by adding the term $\rho$ (description of $\rho$ below), 
and define the following measure.

$$
\widetilde{d \lambda_{x_{0}}}=\left[1+2 \sum_{k=1}^{n-1}\left(A_{k} \cos (k \theta)+i B_{k} \sin (k \theta)\right)+\rho\right] \frac{d \theta}{2 \pi}
$$

The purpose of $\rho$ is to add positive weight to

$$
\left[1+2 \sum_{k=1}^{n-1} \frac{n-k}{n} \cos (k \theta)\right] \frac{d \theta}{2 \pi}
$$

at $\theta=\frac{2 \pi k}{n}$ for $k=1,2, \ldots, n-1$ while it maintains the above three conditions (a)-(c). Now we will show how to choose a desirable $\rho$. Let $\delta=\frac{\pi}{4 n}$. Then when $\theta$ is $\delta$ away from each zero, measure (2.9) is strictly positive. In other words, if $\frac{2 \pi}{n}>\left|\theta-\frac{2 \pi k}{n}\right| \geq \delta$,

$$
\left[1+2 \sum_{k=1}^{n-1} \frac{n-k}{n} \cos (k \theta)\right]>\eta_{k}
$$

for some $\eta_{k}>0$ for each $k=1,2, \ldots, n-1$. Let $\eta=\min \left\{\eta_{k}\right\}_{k=1}^{n-1}$, and define $\rho=\frac{\eta}{2} \cos (2 n \theta)$. Then $\rho$ has period of $\frac{\pi}{n}$ and attains its maximum value $\frac{\eta}{2}$ at $\theta=\frac{m \pi}{n}, m \in \mathbb{Z}$. Notice when $m$ is an even number less than $2 n, \theta=\frac{m \pi}{n}$ are the zeros of $1+2 \sum_{k=1}^{n-1} \frac{n-k}{n} \cos (k \theta)$. Hence as promised, $\rho$ adds positive weight $\frac{\eta}{2}$ to (2.9) at $\theta=\frac{2 \pi k}{n}$ for $k=1,2, \ldots, n-1$. That is, if $\left|\theta-\frac{2 \pi k}{n}\right|<\delta$, then

$$
\left[1+2 \sum_{k=1}^{n-1} \frac{n-k}{n} \cos (k \theta)+\rho\right] \frac{d \theta}{2 \pi}>0 .
$$

Lastly, we must verify that $\left[1+2 \sum_{k=1}^{n-1} \frac{n-k}{n} \cos (k \theta)+\rho\right] \frac{d \theta}{2 \pi}$ remains strictly positive even when $\rho$ is negative. Again $\rho=\frac{\eta}{2} \cos (2 n \theta)$ has period of $\frac{\pi}{n}$ and attains its minimum value $-\frac{\eta}{2}$ at $\theta=\frac{\pi(2 m-1)}{2 n}, m \in \mathbb{Z}$. Starting at some angle $\omega$ where $\rho$ attains its maximum value $\frac{\eta}{2}, \stackrel{2 n}{\rho}=\frac{\eta}{2} \cos (2 n \omega)=\frac{\eta}{2}, \rho$ decreases and passes through its zero, $\rho=\frac{\eta}{2} \cos \left(2 n \omega+\frac{\pi}{4 n}\right)=0$ then its minimum value $\rho=\frac{\eta}{2} \cos \left(2 n \omega+\frac{\pi}{2 n}\right)=-\frac{\eta}{2}$. Recall that we chose $\delta=\frac{\pi}{4 n}$ and result (2.10). Therefore when $\theta$ is at least $\frac{\pi}{4 n}$ away from the zeros of $\left[1+2 \sum_{k=1}^{n-1} \frac{n-k}{n} \cos (k \theta)\right] \frac{d \theta}{2 \pi}$, $1+2 \sum_{k=1}^{n-1} \frac{n-k}{n} \cos (k \theta)$ is guaranteed to be more than $\eta$ while the smallest value of $\rho$ is $-\frac{\eta}{2}$; that is, if $\frac{\pi}{n}>\left|\theta-\frac{2 \pi k}{n}\right| \geq \delta$, then

$$
1+2 \sum_{k=1}^{n-1} \frac{n-k}{n} \cos (k \theta)+\rho>\eta-\frac{\eta}{2}>0 .
$$

Hence,

$$
\lim _{x_{0} \rightarrow 1} \widetilde{d \lambda_{x_{0}}}=\left[1+2 \sum_{k=1}^{n-1} \frac{n-k}{n} \cos (k \theta)+\rho\right] \frac{d \theta}{2 \pi}
$$

where (2.11) is a probability measure over $\Gamma$. Moreover, since the limit of $\widetilde{d \lambda_{x_{0}}}$ is strictly positive, there exists $x_{*}$ near $x_{0}=1$ such that $\widetilde{d \lambda_{x_{*}}} \geq 0$. 
Therefore,

$$
\frac{\left(1-x_{*} z\right)^{n}}{(1-z)^{q}}=\epsilon \int_{\Gamma \times\{1\}} \frac{(1-x z)^{n}}{(1-z)^{q}} d \mu_{1}+(1-\epsilon) \int_{\Gamma \times(\Gamma \backslash\{1\})} \frac{(1-x z)^{n}}{(1-y z)^{q}} \widetilde{d \lambda_{x_{*}}} \times d \nu
$$

where $\mu_{1}, \nu$ and $\widetilde{\lambda_{x_{*}}}$ have been completely identified and equation (2.4) holds. This proves the existence of non extreme points; $\frac{\left(1-x_{*} z\right)^{n}}{(1-z)^{q}}$ where $x_{*}$ is near 1 .

This concludes the proof of Theorem 1.1. The motivation of this paper was to show the existence of non extreme points near $x=1$ which was never shown before. At the moment, we can only achieve this by restricting $p$ to be a positive integer but surprisingly no relation to the size of $q$ except $q \geq 1$. If we are only interested in showing $x=1$ is not extreme, then there are more non extreme points where $p$ no longer has to be a positive integer. It is well-known that $\frac{(1-x z)^{p}}{(1-z)^{q}}$ is a non extreme point when $x=1$ for any $p$ and $q$, provided $q \geq p$. Our recent work on identifying non extreme points for $q \geq 1$, independent of $\mathrm{p}$ does not require $p$ to be a positive integer. However since this particular argument could not be extended to show the existence of non extreme points near $x=1$, I did not include this result in this paper. But perhaps this result together with some special cases will be addressed in a planned companion paper.

Lastly, of course the ultimate goal is to precisely determine all non extreme points for any $p$ and $q$. Combining the results from [3] and Theorem 1.1 in this paper, we pose the following conjecture.

Conjecture 2.3. For fixed $p$ and $q$ with $x=e^{i \theta}$, there exists an angle $\tilde{\theta}=\tilde{\theta}(p, q)$ separating extreme points from non extreme points as follows:

(a) If $0<p \leq 1$, then $\frac{(1-x z)^{p}}{(1-z)^{q}}$ is extreme if $x \neq 1$, and $\frac{(1-x z)^{p}}{(1-z)^{q}}$ is not extreme if $x=1$.

(b) If $p>1$, then $0<\tilde{\theta}<\pi(p-1) /(p+1)$ and $\frac{(1-x z)^{p}}{(1-z)^{q}}$ is extreme if $\tilde{\theta} \leq|\theta| \leq \pi$, and $\frac{(1-x z)^{p}}{(1-z)^{q}}$ is not extreme if $0 \leq|\theta|<\tilde{\theta}$.

In the special case $p=2, q=1$, further analysis suggests that the critical angle $\tilde{\theta}$ should yield an extreme point. According to (v) in Section 1 , for $0<p \leq 1 \frac{(1-x z)^{p}}{(1-z)^{q}}$ is an extreme point if $x \neq 1$, independent of $q$. It is also well-known that $\frac{(1-x z)^{p}}{(1-z)^{q}}$ is a non extreme point when $x=1$ for any $p$ and $q$, provided $q \geq p$. Thus part (a) of Conjecture 2.3 is correct if $0<p \leq 1$ and $q \geq p$. In this paper, we showed that $\frac{(1-x z)^{p}}{(1-z)^{q}}$ is a non extreme point if $q \geq 1$ and $p=n=2,3,4, \ldots$, not only at $x=1$ but also for values of $x$ near 1 . Paired with the results from [3] we thus have extreme points corresponding to values of $x$ beyond the turning points and non-extreme points in intervals near the origin, providing strong evidence for the validity of part (b) of Conjecture 2.3, at least in the more tractable case of integer valued $p$.

\section{References}

[1] D. Aharonov and S. Friedland, On functions of bounded boundary rotation, Ann. Acad. Sci. Fenn. A I Math 585 (1974), 3-18. 
[2] L. Brickman, T. H. MacGregor, and D. R. Wilken, Convex hulls of some classical families of univalent functions, Trans. Amer. Math. Soc 156 (1971), 91-107.

[3] K. Dow and D.R. Wilken, Extreme points of integral families of analytic functions, J. Aust. Math. Soc. 94 (2) (2013), 202-221.

[4] T. H. MacGregor and D. R. Wilken, Handbook of Complex Analysis: Geometric Function Theory, Volume 1, North Holland, Amsterdam, 2002.

[5] S. Perera, Support points and extreme points of some classes of analytic functions, Ph.D. thesis, State University of New York at Albany, 1983.

K. Dow

230 Porter Ave,

Buffalo NY 14201,

United States

dowk@dyc.edu 